\title{
Child with Guillain-Barré Syndrome Responding to Plasmapheresis: A Case Report
}

\author{
Sarmad Al Hamdani ${ }^{a}$ Fatema Yusuf Aljanabi $^{\text {b }} \quad$ Maryam Isa Abdulrasool $^{\mathrm{b}}$ \\ Alaa Haitham Salman ${ }^{b}$ \\ aDepartment of Pediatrics, Dubai Hospital, Dubai Health Authority, Dubai, UAE; \\ ${ }^{b}$ Dubai Medical College, Dubai, UAE
}

\section{Keywords}

Plasmapheresis · Intravenous immunoglobulin · Guillain-Barré syndrome

\begin{abstract}
Intravenous immunoglobulin (IVIG) has long been regarded as the first-line treatment for Guillain-Barré syndrome (GBS), with plasmapheresis only being reserved for severe cases or used as an additional therapy of unproven efficacy. Here, we present the case of a 9-year-old girl with acute motor axonal neuropathy (AMAN), a rapidly progressive subtype of GBS that caused her to fall into respiratory failure. The patient failed to show a response 10 days after starting IVIG, but showed rather quick improvement with plasmapheresis. She received a total of 5 sessions of plasmapheresis on alternate days over a course of 8 days. Before starting plasmapheresis, her muscle strength was $2 / 5$ in both upper limbs and $1 / 5$ in both lower limbs, and she was dependent on mechanical ventilation. Following the first session, her power improved from $2 / 5$ to $4 / 5$ in the upper limbs, and the gag and sucking reflexes were recovered. On day 3 , after the second session was initiated, she was extubated successfully (having been on a ventilator for 2 weeks) and remained on continuous positive airway pressure for the next $48 \mathrm{~h}$, after which she was on room air. In addition, she was having hypertension from the first day of the diagnosis (which was due to autonomic instability), which improved after clonidine to maintain her blood pressure. She was also initially having urinary retention, then was off Foley's catheter. The patient was discharged from the hospital 2 weeks following the first session of
\end{abstract}


plasmapheresis, with power grade $4 / 5$ in both her upper and lower limbs. Her cranial nerves had recovered fully, and she was able to walk with aids.

\section{Background}

Guillain-Barré syndrome (GBS) is a rare neurological disorder in which the body's immune system mistakenly attacks part of its peripheral nervous system due to cross-reactive epitopes (molecular mimicry). It is often triggered by an antecedent infection, most commonly a preceding viral or bacterial infection with organisms such as Campylobacter jejuni, cytomegalovirus, Epstein-Barr virus, or Mycoplasma pneumoniae. The end result is an acute polyneuropathy. GBS is the most common cause of acute flaccid paralysis in healthy infants and children. Most patients with acute inflammatory demyelinating polyradiculopathy (AIDP), the most common form of GBS, develop neurologic symptoms 2-4 weeks after having what initially appears to be a benign febrile respiratory or gastrointestinal infection. Acute motor axonal neuropathy (AMAN) is a pure motor form of GBS. This disorder is distinguished from AIDP by its involvement of predominantly motor nerves and an electrophysiological pattern suggesting axonal damage.

The most common symptoms at presentation in children are pain and gait difficulty. Symmetric or modestly asymmetric lower-extremity weakness may ascend over hours to days to involve the arms, and the muscles of respiration in severe cases. The facial nerve is occasionally affected, resulting in facial weakness. Autonomic dysfunction occurs in approximately one-half of children. Most patients reach their clinical nadir within 2-4 weeks, with subsequent return of function over the course of weeks to months. The initial diagnosis of GBS is based upon the clinical presentation, which is supported by the finding of cerebrospinal fluid (CSF) albuminocytologic dissociation, characterized by an elevated CSF protein level ( $>45$ $\mathrm{mg} / \mathrm{dL}$ ) with a normal CSF white blood cell count [1]. The main modalities of therapy for GBS are intravenous immunoglobulin (IVIG) and plasmapheresis, in addition to supportive management. What treatment to give patients is very controversial, with different opinions and recommendations given by various specialists, depending on the clinical impression and the availability of resources in the setting.

The current ASFA (American Society for Apheresis) guidelines for the use of plasmapheresis as a first-line treatment in AIDP are as follows:

- $\quad$ ASFA Category - I (disorders for which apheresis is accepted as first-line therapy, either as a primary standalone treatment or in conjunction with other modes of treatment)

- ASFA Recommendation Grade - 1A (strong recommendation, high-quality evidence, can apply to most patients in most circumstances without reservation)

An international RCT compared therapeutic plasma exchange (TPE), IVIG, and TPE followed by IVIG in 383 adult patients with severe AIDP and found all three modalities to be equivalent (Plasma Exchange/Sandoglobulin Guillain-Barré Syndrome Trial Group, 1997). Since IVIG is readily available and a more convenient form of immunomodulatory treatment, it is frequently used as initial therapy; the typical dose is $0.4 \mathrm{~g} / \mathrm{kg}$ for 5 consecutive days. Currently there are insufficient data to conclude on the efficacy of TPE after IVIG failure [2]. 


\section{Case Presentation}

A 9-year-old previously healthy girl presented with a history of headache, dizziness, and visual disturbances for 2 days followed by inability to bear weight for 1 day. The headache was frontal and continuous, associated with dizziness and vertigo. She also had pain in her neck, back, and lower limbs and 3 episodes of nonprojectile nonbilious vomiting on the first day of the illness. On the day before admission, she had begun having progressive, bilateral leg weakness and was unable to walk or sleep properly that night. Next morning, she could not get out of bed, so her parents brought her to our hospital. She had not passed urine in 12 $\mathrm{h}$ and the last bowel movement had been 4 days prior to admission. There was no fever, sore throat, cough, or runny nose and no loose stools. Nor was there a history suggestive of trauma, toxin ingestion, traveling, or exposure to sick contacts.

Two weeks previously, the patient had been diagnosed with otitis media and treated with antibiotics at a private clinic. She had been afebrile during that illness. During the current encounter, the patient was admitted to the high dependency unit of our hospital at 6:45 a.m. under close monitoring. On examination at admission, she was drowsy but reusable and oriented to time, place, and person. Her speech was sluggish. There was no facial asymmetry. She was not in distress but had shallow breathing. Her respiratory rate was 25 breaths/min, with oxygen saturation $>94 \%$ on $1 \mathrm{~L} / \mathrm{min}_{2}$ via a nasal cannula. She had enlarged, uncongested tonsils. Her extraocular movements were normal. Both pupils were $4-5 \mathrm{~mm}$ in size and reactive to light. She was complaining of pain on movement of the extremities. Gait could not be assessed as the patient refused to stand. She could only sit with support. Her upper limbs had a normal tone, with power grade 3/5 and normal reflexes. Her lower limbs were hypotonic, with power grade $2 / 5$, and diminished reflexes bilaterally. The plantar reflexes were going down. Her cerebellar signs were inconclusive as the patient was drowsy and tired. There were no petechial rashes and no meningeal signs.

The child's sensorium remained intact until 4:30 p.m. on the same day, when she went into respiratory failure. A crash call was announced, and the patient was intubated and shifted to the intensive care unit (ICU). Upon repeat examination in the ICU, there were bilateral 6th cranial nerve palsies, partial 3rd nerve palsy, and bilateral facial weakness. Speech was sluggish and the gag reflex was absent. Her strength was $2 / 5$ in both upper limbs, and $1 / 5$ in both lower limbs. Reflexes were just elicitable in the upper limbs but were absent in both lower limbs. No sensory level was detected. Respiratory examination showed weak respiratory efforts, requiring oxygen support to maintain her saturation. On examination of her abdomen, the bladder was distended; therefore, a Foley catheter was inserted.

Considering the acute progression of her ascending weakness, and bowel and bladder involvement with examination findings of 3rd, 4th, 6th, 7th, 9th, and 10th cranial nerve palsies, a diagnosis of GBS seemed highly likely. A subsequent nerve conduction study done in the first week was suggestive of AMAN. Lumbar puncture was also done, which turned out normal. She underwent brain and whole-spine MRI (all resulted normal, thereby excluding the possibility of transverse myelitis). Her autoimmune profile was sent on (for myasthenia gravis, systemic lupus erythematosus, and autoimmune thyroiditis), which came out normal. The patient tested positive for ganglioside GD1a and GD1b antibodies (very suggestive of AMAN) and negative for GM1, GM2, and GQ1b antibodies (making Miller-Fisher syndrome unlikely).

She had been started on triple antibiotics upon admission for suspected CNS infection, which were stopped after the results of the lumbar puncture and nerve conduction study were out, and with the presence of ganglioside GD1a and GD1b antibodies, further supporting the final diagnosis of AMAN, a subtype of GBS. During her stay in the ICU, the patient was initiated 
on ventilator support and started on IVIG therapy for 5 days at a dose of $2 \mathrm{~g} / \mathrm{kg}$. She was started on clonidine in view of persistently high blood pressure readings attributed to the autonomic instability in GBS. She was receiving tube feeding and physiotherapy and was given Clexane injections of $0.2 \mathrm{~mL}$ ( $20 \mathrm{mg}$ total) subcutaneously daily from day 5 of admission as part of deep vein thrombosis prophylaxis, but in view of the persistent hematuria, Clexane was stopped and deep vein thrombosis stockings were prescribed.

A randomized study revealed that treatment with $2 \mathrm{~g} / \mathrm{kg}$ body weight IVIG within 2 days did not yield better results than distributing the same total dose over 5 days. The two schedules were equally tolerated, with only mild transient side effects. Early relapses occurred more frequently with the 2-day than with the 5-day regimen [3].

Over the following 10 days, there was only mild improvement in power in the upper limbs (grade 2). Extubation was tried twice, which the patient failed on both occasions. In view of no further improvement, plasmapheresis was started after 10 days of IVIG and supportive care. The patient received 5 cycles of plasmapheresis on alternate days over a course of 8 days. Before the first 2 sessions of plasmapheresis, the following tests were ordered for the patient: full blood count, prothrombin time, partial thromboplastin time, fibrinogen, D-dimer, immunoglobulin level, urea and electrolytes, calcium, phosphate, and magnesium. In addition, serum calcium, albumin, and the coagulation profile were checked after all the sessions. For the first session, the replacement fluid was $900 \mathrm{~mL}$ fresh frozen plasma and albumin 5\% at 1,000 $\mathrm{mL}$, which were given through a hemodialysis (HD) catheter (11.5 Fr). For the remaining sessions, we used 1,750 mL fresh frozen plasma and albumin $5 \%$ at $750 \mathrm{~mL}$. The hemodialysis catheter was not used for blood sampling or IV medications and was kept locked with heparin.

Following the first session of plasmapheresis, there was a marked clinical improvement with power in the upper limbs of grade $3 / 5$ (Fig. 1) and presence of the gag and suck reflexes on suctioning. After the second session of plasmapheresis, the patient was able to move her lower limbs. She was weaned from controlled ventilation over the next 2 days and was kept on continuous positive airway pressure support for $48 \mathrm{~h}$ before being extubated to room air. Following the third dose of plasmapheresis, the patient was shifted to the general pediatric ward from the ICU. She was unable to pass urine, so she was catheterized again and started bladder training with clamping the Foley catheter. The child was in pain, so tramadol and gabapentin were started, which controlled the pain well. The last 2 sessions of plasmapheresis were given on 2 consecutive days, after which the patient was able to move her head in all directions and was able to swallow both liquids and solids. Power was grade $4 / 5$ in the upper limbs and 3/5 in the lower limbs. Clonidine was gradually tapered and was stopped after her blood pressure readings remained stable throughout. She continued to receive active chest and limb physiotherapy. The patient continued to show clinical improvement and was off the Foley catheter within the next week. Power improved (grade $4 / 5$ in both the upper and lower limbs). Reflexes were elicitable (+1) in both the upper and lower limbs. The cranial nerve palsies recovered, and the patient's eye movements were normal in all directions. The patient needed help sitting from the supine position, but could sit unsupported. She was able to stand with support and to walk short distances using aids. After this 1-month-long stay in the hospital, the patient was discharged home with a follow-up appointment in 6 weeks.

\section{Discussion}

The main purpose of our case report was to evaluate the efficacy of plasmapheresis versus IVIG in the management of GBS, especially in patients with rapidly progressive and 
severely aggressive GBS, who may benefit from other modalities of treatment such as plasmapheresis, which proved to be superior to IVIG in our case. Our patient, who displayed minimal recovery after 10 days of IVIG administration, showed quite striking clinical improvement following plasmapheresis.

One of the differential diagnoses in our case was transverse myelitis, but this was ruled out by the normal MRI results and the absence of a sensory level on physical examination. What further supports a diagnosis of GBS is the result of a nerve conduction study, which, in our patient, was diagnostic of AMAN. Nerve conduction studies allow a diagnosis of pediatric GBS in as many as $90 \%$ of cases during the first week of symptoms [4]. This discounts the normal CSF study, which can be negative (no albuminocytologic dissociation) in one-third to one-half of patients if tested earlier than 1 week after symptom onset [5]. More importantly, our patient tested positive for ganglioside GD1a and GD1b antibodies, which in one study was associated with AMAN in up to $60 \%$ of their patients [6].

\section{Prognosis and Recovery}

The recovery period in pediatric GBS is long, often weeks to months, with a median estimated recovery time of 6-12 months. In one small pediatric series, the median time from onset of symptoms to complete recovery was 73 days. Full recovery within 3-12 months is experienced by $90-95 \%$ of pediatric patients with GBS [7]. Our patient, on the other hand, started recovering immediately after plasmapheresis had been initiated, and she was able to walk with aids 1 month from the start of the disease.

On literature review, we found some reports supporting IVIG, some supporting plasmapheresis/plasma exchange (PE) and some reporting no significant difference between the two, compared to only supportive management [8]. However, the majority of the studies were performed on adults, with limited trials carried out in pediatric patients.

One study recommended the use of IVIG as an initial therapy for pediatric GBS patients, claiming that it has fewer side effects than PE, both having been associated with the same duration of stay in the hospital and the same time till achieving ambulation [9]. A meta-analysis published in 2016 displayed that there is no evidence for any superiority in efficacy or safety of either IVIG or plasmapheresis in the management of GBS [10].

On the other hand, a standard dose of IVIG may not be sufficiently effective in some GBS patients, necessitating a second dose, which will further add to the costs [11]. In addition, PE can still be as effective if initiated up to 4 weeks from the start of the disease, whereas IVIG is better administered within 2 weeks of disease onset. Thus, PE can be used for patients who present later in the disease course [12].

Despite limited evidence, it seems that the prognosis is poorer for GBS patients in the following groups [13-18]: (a) those very young ( $<2$ years); (b) those very weak at presentation; (c) those having cranial nerve involvement; (d) those quadriparetic on day 10; (e) those requiring ventilator support; and (f) those having inexcitable motor nerves on nerve conduction studies.

In general, the therapeutic response following IVIG is good in those with demyelinative but unsatisfactory in those with axonopathic features [19]. One study suggested that GBS patients with axonal involvement, and those with recurrent or familial GBS forms, may be refractory to IVIG treatment and PE should be considered early in these cases [20].

A Cochrane review has shown that PE is better than supportive treatment [12]. Another reviewer concluded that $\mathrm{PE}$ is the first and only treatment that has been proven to be superior 
to supportive treatment alone in GBS [21]. Some recommend using both modalities of treatment, starting with a dose of IVIG, followed by PE in refractory cases that fail to respond, even if, perhaps, starting PE earlier would further shorten the patient's hospital stay and the time spent on a ventilator. There is an ongoing debate about whether IVIG should be used before or after PE if both of these treatment modalities were to be used [22].

\section{Conclusion}

The treatment of GBS is an unresolved issue, and guidelines for the management of both pediatric and adult patients with GBS need to be set up so that healthcare professionals can provide the most effective treatment for their patients without hesitation and hence improve their patients' outcome and long-term prognosis.

This case report may open doors for new research on the efficacy of plasmapheresis and whether it should be used with IVIG or even regarded as a sole treatment for GBS, especially in places where IVIG is unavailable.

\section{Statement of Ethics}

The authors have no ethical conflicts to disclose.

\section{Disclosure Statement}

All authors and their relatives have nothing to disclose; they have no conflict of interest and no financial interest in the subject matter. Written informed consent was obtained from the patient's family. There are no patient identifiers in the case report which may link the patient to the report. The study protocol was approved by the institute's committee on human research.

\section{Funding Sources}

The work for this case report did not receive any funding.

\section{Author Contributions}

S. Al Hamdani took care of the patient, conceived the idea, wrote the case report, extracted and analyzed data, and approved the manuscript. F.Y. Aljanabi, M.I. Abdulrasool, and A.H. Salman conceived the idea, wrote the case report, extracted and analyzed data, and approved the manuscript. 


\section{References}

1 Willison HJ, Jacobs BC, van Doorn PA. Guillain-Barré syndrome. Lancet. 2016 Aug;388(10045):717-27.

2 Padmanabhan A, Connelly-Smith L, Aqui N, Balogun RA, Klingel R, Meyer E, et al. Guidelines on the Use of Therapeutic Apheresis in Clinical Practice - Evidence-Based Approach from the Writing Committee of the American Society for Apheresis: The Eighth Special Issue. J Clin Apher. 2019 Jun;34(3):171-354.

3 Korinthenberg R, Schessl J, Kirschner J, Mönting JS. Intravenously administered immunoglobulin in the treatment of childhood Guillain-Barré syndrome: a randomized trial. Pediatrics. 2005 Jul;116(1):8-14.

4 Delanoe C, Sebire G, Landrieu P, Huault G, Metral S. Acute inflammatory demyelinating polyradiculopathy in children: clinical and electrodiagnostic studies. Ann Neurol. 1998 Sep;44(3):350-6.

5 Dimachkie MM, Barohn RJ. Guillain-Barré syndrome and variants. Neurol Clin. 2013 May;31(2):491-510.

6 Ho TW, Willison HJ, Nachamkin I, Li CY, Veitch J, Ung H, et al. Anti-GD1a antibody is associated with axonal but not demyelinating forms of Guillain-Barré syndrome. Ann Neurol. 1999 Feb;45(2):168-73.

7 DiFazio MP, Patel NC, Patel MN, Chhibber S, Tseng BS. Pediatric Guillain-Barré syndrome. Medscape. 2019 [updated 2019 Nov 14].

8 Hughes RA, Raphaël JC, Swan AV, Doorn PA. Intravenous immunoglobulin for Guillain-Barré syndrome. Cochrane Database Syst Rev. 2004;(1):CD002063.

9 Abd-Allah SA, Jansen PW, Ashwal S, Perkin RM. Intravenous immunoglobulin as therapy for pediatric Guillain-Barré syndrome. J Child Neurol. 1997 Sep;12(6):376-80.

10 Ortiz-Salas P, Velez-Van-Meerbeke A, Galvis-Gomez CA, Rodriguez Q JH. Human Immunoglobulin versus Plasmapheresis in Guillain-Barré Syndrome and Myasthenia Gravis: A Meta-Analysis. J Clin Neuromuscul Dis. 2016 Sep;18(1):1-11.

11 van Doorn PA, Kuitwaard K, Walgaard C, van Koningsveld R, Ruts L, Jacobs BC. IVIG treatment and prognosis in Guillain-Barré syndrome. J Clin Immunol. 2010 May;30(1 Suppl 1):S74-8.

12 Winer JB. Guillain-Barré syndrome. BMJ. 2008 Jul;337:a671.

13 Bradshaw DY, Jones HR Jr. Guillain-Barré syndrome in children: clinical course, electrodiagnosis, and prognosis. Muscle Nerve. 1992 Apr;15(4):500-6.

14 Hung PL, Chang WN, Huang LT, Huang SC, Chang YC, Chang CJ, et al. A clinical and electrophysiologic survey of childhood Guillain-Barré syndrome. Pediatr Neurol. 2004 Feb;30(2):86-91.

15 Barzegar M, Toopchizadeh V, Maher MH, Sadeghi P, Jahanjoo F, Pishgahi A. Predictive factors for achieving independent walking in children with Guillain-Barré syndrome. Pediatr Res. 2017 Aug;82(2):333-9.

16 Cole GF, Matthew DJ. Prognosis in severe Guillain-Barré syndrome. Arch Dis Child. 1987 Mar;62(3):288-91.

17 Vajsar J, Fehlings D, Stephens D. Long-term outcome in children with Guillain-Barré syndrome. J Pediatr. 2003 Mar;142(3):305-9.

18 Lin JJ, Hsia SH, Wang HS, Lyu RK, Chou ML, Hung PC, et al. Clinical variants of Guillain-Barré syndrome in children. Pediatr Neurol. 2012 Aug;47(2):91-6.

19 Hughes RA, Cornblath DR. Guillain-Barré syndrome. Lancet. 2005 Nov;366(9497):1653-66.

20 Buzzigoli SB, Genovesi M, Lambelet P, Logi C, Raffaelli S, Cattano D. Plasmapheresis treatment in GuillainBarré syndrome: potential benefit over intravenous immunoglobulin. Anaesth Intensive Care. 2010 Mar;38(2):387-9.

21 Raphaël JC, Chevret S, Hughes RA, Annane D. Plasma exchange for Guillain-Barré syndrome. Cochrane Database Syst Rev. 2002;(2):CD001798.

22 Hughes RA, Swan AV, van Doorn PA. Intravenous immunoglobulin for Guillain-Barré syndrome. Cochrane Database Syst Rev. 2014 Sep;(9):CD002063.

This work was conducted at the Pediatric ICU, Pediatric Department, Dubai Hospital, Dubai, UAE. 


\section{Response of Guillain Barre Syndrome to plasmapheresis}

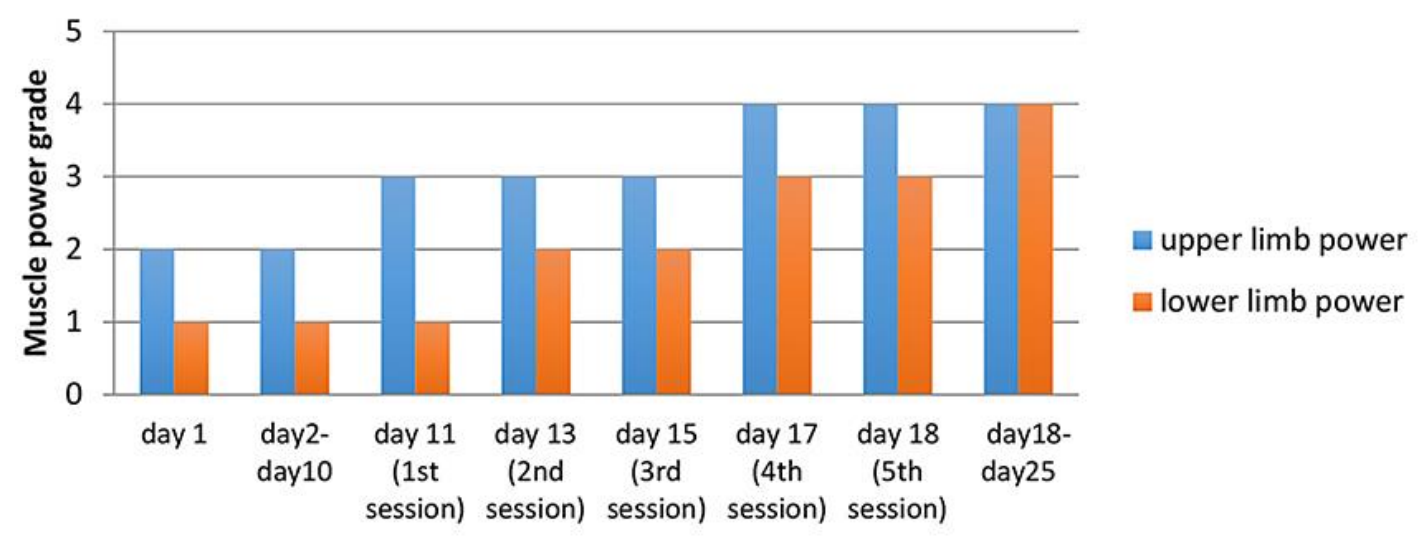

Fig. 1. Clinical course of our patient with Guillain-Barré syndrome before and after starting plasmapheresis. 